\title{
HYSTERESIS AND EDDY CURRENT EXPONENTS FOR SILICON STEEL
}

\author{
BY W. J. WOOLDRIDGE
}

It is my intention to bring before the Institute as briefly as may be, the apparent changes in general direction of curves required for predetermining core losses of apparatus, especially transformers, in which silicon steel is used.

When this alloyed steel first came into use, some years ago, curves were drawn up based on losses found in samples tested at 5,000 $B$ and $10,000 B$ and retaining the use of the Steinmetz exponents which had for many years stood as approximately correct values for commercial electrical sheet steel, viz., eddy current loss increasing as $B^{2}$ and hysteresis loss increasing as $B^{1 \cdot 6}$.

It was quite natural that this material should first be used in transformers both because of the constant demand for lower core losses, the prospect of more compact designs and also because of the mechanical qualities of silicon steel.

With ordinary steel the limit of design for transformers was along the line of heating. That is to say, the limit was the watts per square inch of effective radiating surface.

With the lower watts per pound of the new steel an inrrease in core density above that generally used in the old material seemed allowable and was also desirable in order to offset the higher cost per pound of the steel. Core loss curves made on such transformers showed the material at low densities to be in accord with design curves, but at medium densities $(12,000 \mathrm{~B}$ for instance) the loss was considerably above anticipated results.

In seeking the cause of the trouble the well established exponents which had proven satisfactory for so long, were not at 
first considered. The test curves when carried further, to relatively high densities, showed a continued increase but not logarithmic. It was noted in such cases that the power factor decreased rapidly, this decrease coinciding in a marked degree to

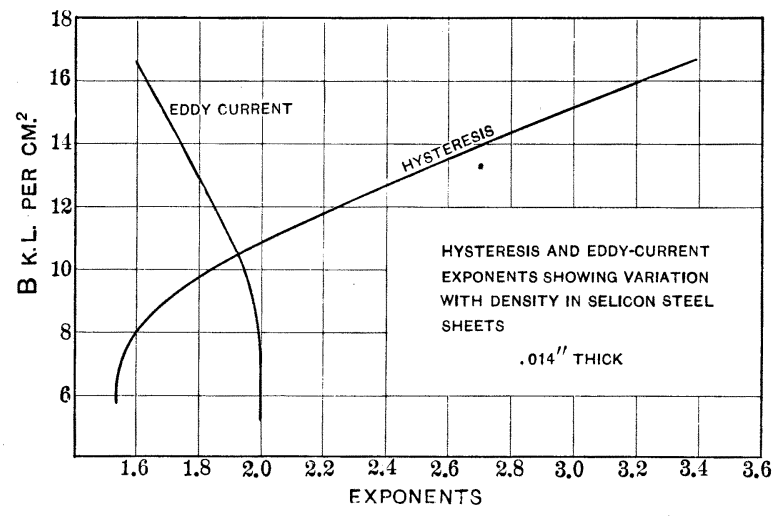

FIG. 1

the increased core loss as shown by the wattmeters. The higher loss was, therefore, assumed to be due at least in part to incorrect wattmeter readings and in part to a possible change in wave form.

(60 CYCLES)

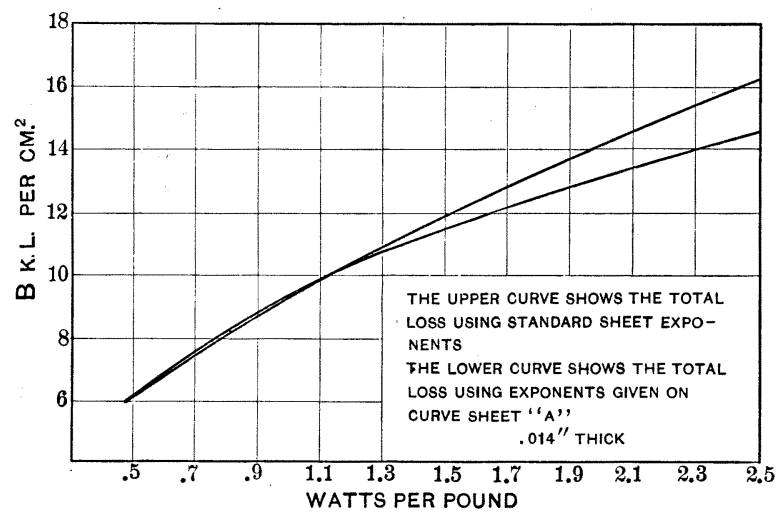

FIG. 2

Later, the development of the iron clad instrument, giving correct readings at low power factors, showed that the premise regarding the effect of incorrect wattmeter readings to be largely a wrong one. Tests on a small core carefully tested on sine 
wave and then used in conjunction with the transformer under test, enabling a ready and convenient correction for wave form, gave further evidence that the high core loss at high densities was inherent.

Careful tests on variously proportioned cores, such as rings without gaps, rectangular cores, and complete transformers, were found to agree closely and finally led to the inevitable conclusion that the exponents regularly used for ordinary steel did not hold for silicon steel.

This has been confirmed by several other investigators and has been published in the Bulletin of the Bureau of Standards, and to some extent by the German technical press.

Both hysteresis and eddy current exponents were determined and it was found these were not a constant value for either component, the hysteresis increasing more and more rapidly as the density increased, while the eddy current decreased but to a lesser extent. The average values found are shown in the curves Fig. 1. In transformers, the decreasing eddy current loss does not materially offset the increasing hysteresis from the fact that the eddy current loss in thin sheets is such a small proportion of the total.

Fig. 2 is given to show the relation between two curves, the upper one of which is plotted using the old ordinary steel exponents and the lower curve being plotted using the exponents shown in Fig. 1, assuming the same values at 10,000 $B$.

The values are based on tests made on 0.014 -in. sheet steel obtained from several steel makers in this country and abroad.

It is interesting to note that the hysteresis exponent in the neighborhood of $16,000 B$ is more than double the old value as used for ordinary steel. 